\title{
STUDY OF THE INTEGRATION OF LIDAR AND PHOTOGRAMMETRIC DATASETS BY IN SITU CAMERA CALIBRATION AND INTEGRATED SENSOR ORIENTATION
}

\author{
Mitishita, E ${ }^{*}$., Costa, F., Martins, M. \\ Geodetic Sciences Graduate Program - Department of Geomatics - Federal University of Paraná, UFPR - Centro Politécnico - Setor \\ de Ciências da Terra - CEP 81.531-990 - Curitiba, Paraná, Brazil - mitishita@ufpr.br; felipe@dsg.eb.mil.br; \\ marlo_martins@hotmail.com
}

Commission I

KEY WORDS: Integrated Sensor Orientation, Direct Sensor Orientation, In situ Calibration, Photogrammetry, Lidar.

\begin{abstract}
:
Photogrammetric and Lidar datasets should be in the same mapping or geodetic frame to be used simultaneously in an engineering project. Nowadays direct sensor orientation is a common procedure used in simultaneous photogrammetric and Lidar surveys. Although the direct sensor orientation technologies provide a high degree of automation process due to the GNSS/INS technologies, the accuracies of the results obtained from the photogrammetric and Lidar surveys are dependent on the quality of a group of parameters that models accurately the user conditions of the system at the moment the job is performed. This paper shows the study that was performed to verify the importance of the in situ camera calibration and Integrated Sensor Orientation without control points to increase the accuracies of the photogrammetric and LIDAR datasets integration. The horizontal and vertical accuracies of photogrammetric and Lidar datasets integration by photogrammetric procedure improved significantly when the Integrated Sensor Orientation (ISO) approach was performed using Interior Orientation Parameter (IOP) values estimated from the in situ camera calibration. The horizontal and vertical accuracies, estimated by the Root Mean Square Error (RMSE) of the 3D discrepancies from the Lidar check points, increased around of $37 \%$ and $198 \%$ respectively.
\end{abstract}

\section{INTRODUCTION}

An important prerequisite for using the photogrammetric and Lidar datasets simultaneously is having both data in the same mapping or geodetic frame. Indirect and direct georeferencing are the procedures used to perform this datasets integration by the estimation of the Exterior Orientation Parameters (EOP) of the images of photogrammetric block. Indirect georeferencing uses a conventional bundle adjustment and Lidar data as a control of position information. For this approach, methods to extract geometric primitives, such as points, lines and areas, are required because the Lidar point cloud does not directly mapping these geometric features. Several studies were conducted on this thematic, such as Habib et al., 2004; Delara et al., 2004; Habib et al., 2005; Mitishita et al., 2008; Wildan et al., 2011.

Nowadays direct sensor orientation is a common procedure used in simultaneous photogrammetric and Lidar surveys. This procedure can automatically acquire the LIDAR and imagery data sets in the same geodetic or mapping frame. Although the direct sensor orientation technologies provide a high degree of automation process due to the GNSS/INS technologies, the accuracies of the obtained results from the photogrammetric and Lidar surveys are dependent on the quality of a group of parameters that models accurately the user conditions of the system at the moment the job is performed. Although the LIDAR and image sensors share the same inertial measurement unit (IMU), frequently the data sets do not match accurately due to system calibration failures. Usually, the photogrammetric system calibration is performed independently of the LIDAR system calibration and direct georeferencing of images depends on the local flight conditions, for example, temperature and atmospheric pressure variability may modify the relative position and orientation of the camera relative to IMU
(Kersting, 2011). Additionally, the atmospheric refraction may modify the collinearity condition of the light ray in its trajectory between object and image spaces (Andrade 1977).

Many researchers (Jacobsen, 2000; Heipke et al., 2002; Cramer and Stallman, 2002; Wegmann, H., 2002; Honkavaara et al., 2003) investigated the stability of the geometric relationship of the IMU to the imaging system and the stability of the interior orientation. They concluded that the interior orientation and the mounting parameters can vary over time. For instance, the interior orientation parameters can change under flight conditions due to the effects of temperature and pressure (Yastikli and Jacobsen, 2005). Then, to improve the accuracy of the Direct Sensor Orientation, the system calibration, including the interior orientation and mounting parameters, is recommended before or after each photogrammetric mission. The system calibration, in principle, can be performed before or after every mission to check the quality of the mounting and IOP. However, due to technical requirements, time and cost, the system calibration is not regularly applied, resulting inaccuracies in the photogrammetric and Lidar datasets integration.

Thus, using Lidar data as a control of position information, the Integrated Sensor Orientation (ISO) can used to improve the quality of the photogrammetric and Lidar datasets integration by the refinement of the direct EOP estimation when its values do not attain the required accuracies. However, to carry out the ISO with a minimum number of known ground control points over the block of images area or without any use of ground control points, three basic conditions must be met. First, the block configuration should have enough forward and side overlap areas with a minimum number of tie points in these areas (Von Gruber positions); second, accurate IOP values and third, 
accurate standard deviation for the direct EOP values (Cramer and Stallman, 2001).

Considering the aforementioned, this paper shows the study that was performed to verify the importance of the in situ camera calibration and Integrated Sensor Orientation to increase the accuracies of the photogrammetric and LIDAR datasets integration. The Lidar dataset is used as a control of position information for the photogrammetric survey performed by the direct sensor orientation technology.

\section{MATERIALS AND METHODOLOGY}

\subsection{Photogrammetric and Lidar surveys}

The TRIMBLE digital aerial camera AIC PRO +65 , mounted with HR Digaron-W $50 \mathrm{f} / 1.4$ lens was used in this study. The camera's CCD sensor has 60 million effective pixels $(8984 \times$ 6732 pixels), dimension of $53.9 \mathrm{~mm} \times 40.4 \mathrm{~mm}$ and the pixel size equal to $0.006 \mathrm{~mm}$. The TRIMBLE camera was connected to the Optech Airborne Laser Scanner ALTM_Pegasus_HD500 to perform airborne Lidar and imagery surveys simultaneously. The camera was installed on the same platform as the Lidar system. It was physically connected to the Lidar system through an RS232 serial cable to record the instants that the images are taken along the Lidar GNSS/INS trajectory in order to compute the position and orientation of the camera. The Optech Airborne Laser Scanner Pegasus_HD500 has an applanix IMU POS AV 510. The IMU absolute accuracies (RMS) - Position < $0.1 \mathrm{~m}$; Roll and Pitch $<0.005$ deg; Yaw $<0.008$ deg.

A photogrammetric image block was acquired on August, 2012. The block has six strips, taken in opposite directions (approximately north-to-south and south-to-north) with around $45 \%$ of lateral overlap. Each strip has sixteen images, acquired with nearly $60 \%$ forward overlap. Figure 1 shows the layout of image block. For the applied flight height of approximately $1600 \mathrm{~m}$, the image pixel resolution on the ground (GSD) resulted close to $0.18 \mathrm{~m}$. A suburban area approximately $57 \mathrm{~km}^{2}$ in size, of the city Curitiba (State of Paraná - Brazil) was covered by the images. Simultaneously to the photogrammetric survey, the individual Lidar strips were collected with a mean point density of 5 points $/ \mathrm{m}^{2}$ (nearly $0.25 \mathrm{~m}$ point spacing). According to the sensor and flight specifications, $0.18 \mathrm{~m}$ horizontal and $0.15 \mathrm{~m}$ vertical accuracies are expected for the acquired Lidar data.

\subsection{Lidar control points (LCPs) extraction}

Thirty-seven Lidar Control Points are used in this study. Only three LCPs are used, as the control points, to perform the in situ camera calibration. The remained LCPs are used as check points to verify the horizontal and vertical accuracies of the photogrammetric and Lidar datasets integration. There are a large number of approaches developed to extract the 3D object coordinates of the image point feature using Lidar point cloud. The approach, used in this study, focused on a semi-automatic point feature extraction by intersection of three building roof planes. According to Costa et al., (2017) the used approach is performed in four steps: filtering Lidar points on the building of roofs; roof building planes extraction; roof building planes modeling; three planes intersection (LCP Characterization).

\subsection{In situ camera calibration}

The in situ camera calibration is used to estimate the interior orientation parameters of the TRIMBLE camera in the flight conditions. Using the collinearity equations and Least Squares Bundle Block Adjustment (BBA), the theoretical collinearity condition among the point image, camera exposition station and point object was in practice recovered by additional parameters related to lens distortions, coordinates of principal point and the sensor distortion. In this study, the in situ camera calibration uses Lidar Derived Control Points (LCPs) and a small sub-block of images extracted from the entire image block obtained in the aerial survey. This approach does not require a calibration test field and also neither traditional ground control points.

The sub block has two strips taken in opposite directions (approximately north-to-south and south-to-north) with around $45 \%$ of lateral overlap. Each strip has three images, acquired with nearly $60 \%$ forward overlap. Three LCPs were used as ground control points. According to Mitishita et al., (2016), three non-aligned LCPS are required to perform the in situ camera calibration process considering the sub-block dimension. Approximately 26 tie points, close to Von Gruber regions, were measured manually, using the Leica Photogrammetry Suite (LPS) 2011. Additionally, the values of the images' EOP from direct sensor orientation are included in the bundle adjustment as additional observations by weight constraint. The EOP values are weighted according to the nominal accuracies. Figure 1 also shows the spatial distribution of three LCPs and the position of the sub-block in the entire images block.

\subsection{Integrated sensor orientation without control points}

The Integrated Sensor Orientation is performed to refine the values of images' exterior orientation parameters (EOP) estimated by direct sensor orientation. It considers that ISO, using IOP values estimated under flight conditions, can improve the accuracies of photogrammetric and Lidar datasets integration.

The new IOP values, due to the high correlation among parameters in the bundle adjustment, can model a parcel of inaccuracies of the direct determination of the EOP and the displacement of the collinearity condition. Considering this supposition, the traditional in situ calibration of the imaging sensor using the physical model, as proposed by Brown, (1971) is used to compute a new set of the Interior Orientation Parameters.

Two ISO experiments are performed. The first uses the IOP provided by the photogrammetric company (camera manufacturer certificate) and in the second one, the IOP, estimated by the in situ camera calibration, were used. The values of EOPs from the direct sensor orientation were included in the bundle adjustment as additional observations by weight constraint. In the first experiment, the EOP values were weighted according to the nominal precisions. The second experiment used the EOP precisions that were estimated in situ calibration. The UFPR Self Calibration Bundle Adjustment Software was used to perform the ISO experiments.

\section{RESULTS AND DISCUSSIONS}

\subsection{Lidar control points (LCPs) extraction}

Thirty-seven Lidar Control Points (LCPs) were computed using the approach discussed in section 2.2. As mentioned before the accuracies of photogrammetric and Lidar datasets integration are obtained by comparison of the 3D coordinates of the LCPs, computed by photogrammetric intersection, to 3D coordinates, computed by three roof planes intersection. For this verification, thirty-seven LCPs, used as check points, are distributed in the entire images block, as can be seen in Figure 1. 


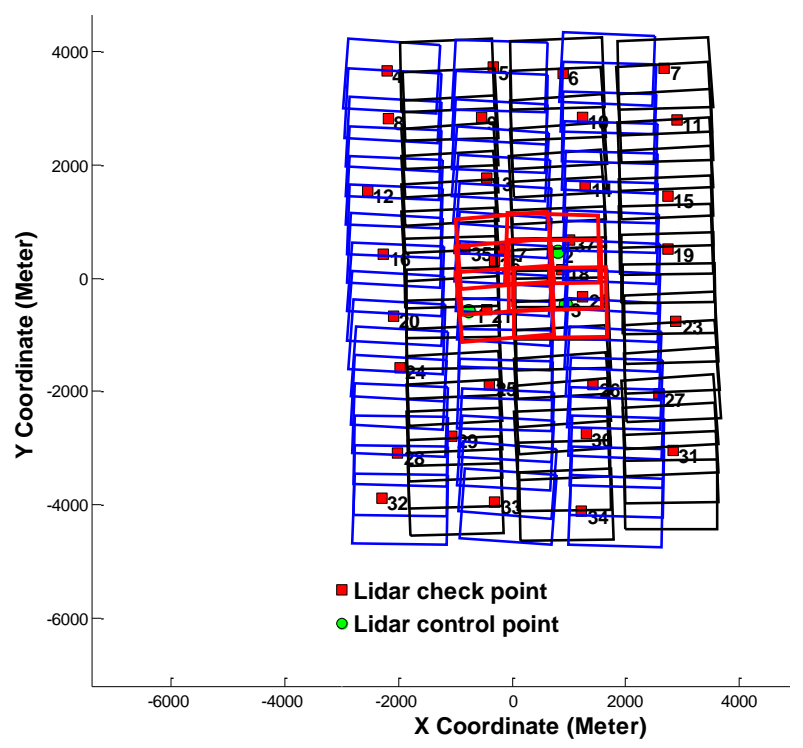

Figure 1. Layouts of the entire images block and the sub-block (red lines) and spatial distribution of the 37 LCPs.

\subsection{In situ Camera Calibration}

The in situ camera calibration was performed using the following measurements precisions: $0.003 \mathrm{~mm}$ (half of pixel) for $\mathrm{x}$ and $\mathrm{y}$ image coordinates; $18 \mathrm{~cm}$ for $\mathrm{X}$ and $\mathrm{Y}$ coordinates and $15 \mathrm{~cm}$ for $\mathrm{Z}$ coordinates for the LCPs (Lidar 3D coordinates accuracies); for the direct EOPs, nominal values were adopted 10 centimeters for positions and 18 seconds for Omega and Phi and 29 seconds for Kappa, considering the results of the trajectory accuracies in Post-Processing mode.

The UFPR Bundle Block Adjustment (calibration and phototriangulation) is used to perform the in situ camera calibration experiment. Table 2 shows the values of IOP that were considered significant. A parameter was considered significant when its standard deviation (parameter's precision estimation), obtained from the variance-covariance matrix, was at least ten times smaller than the parameter magnitude. The values of the nominal IOP from the camera certificate were also included in Table 2 to support the discussion and analyses.

The precision analysis of the in situ camera calibration experiment, performed through the inspection of the values of the root mean square errors of the measurement residuals reported in Table 1, reveals that the systematic errors in the measurements were properly modeled by the parameters estimated in the in situ camera calibration procedure.

\begin{tabular}{|c|c|c|c|}
\hline \multicolumn{4}{|c|}{ Residuals in image coordinates (microns) } \\
\hline RMSE / Max & $x=2 / 6$ & & $2 / 4$ \\
\hline \multicolumn{4}{|c|}{ Residuals in Lidar Control Points coordinates (centimeters) } \\
\hline RMSE / Max & $\mathbf{X}=14 / 19$ & $\mathbf{Y}=9 / 12$ & $\mathbf{Z}=6 / 8$ \\
\hline \multicolumn{4}{|c|}{ Residuals in camera station's coordinates (centimeters) } \\
\hline RMSE / Max & $\mathbf{X s}=2 / 3$ & $\mathbf{Y s}=1 / 1$ & $\mathbf{Z s}=14 / 21$ \\
\hline \multicolumn{4}{|c|}{ Residuals in camera station's orientation (seconds) } \\
\hline RMSE / Max & $\omega=11 / 14$ & $\varphi=4 / 7$ & $\chi=7 / 11$ \\
\hline \multicolumn{4}{|c|}{$(\boldsymbol{\sigma} \boldsymbol{0})=$ Posteriori variance of unit weight $=0.779$} \\
\hline & $\begin{array}{l}\text { ISE }=\text { Root } \mathrm{m} \\
\text { Max = Maxin }\end{array}$ & $\begin{array}{l}\text { square err } \\
\text { residuals; }\end{array}$ & \\
\hline
\end{tabular}

Table 1. Main Results of the Residuals Analysis Performed In Situ Camera Calibration
The RMSE of the residuals from the images measurements are smaller than the a-priori precisions adopted. Only three photogrammetric points have residuals larger than half of the pixel size and the RMSE in the Lidar Control Points coordinates are close to the a-priori precision. Additionally, the RMSE of the residuals of the direct estimation of the positions (Xs, Ys and $\mathrm{Zs})$ and orientations $(\omega, \varphi, \chi)$ of the image block are approximately three times smaller than the a-priori adopted precision. Except in the $\mathrm{Z}$ coordinate of the camera stations, the RMSE is close to the adopted precision.

These aforementioned results and the precisions of the IOP, shown in Table 2, confirm that the in situ camera calibration achieved acceptable precisions. Comparing the IOP from the camera manufacturer certificate to the IOP from in situ camera calibration, shown in Table 2, it can be seen that the focal length and the principal point coordinates changed their values significantly. The variations might be attributed to instability in the camera geometry and/or environmental changes under operational conditions or due the mathematical correlation among parameters ( $\mathrm{f}-\mathrm{Zs} ; \mathrm{x}_{\mathrm{o}}-\mathrm{Xs} ; \mathrm{y}_{\mathrm{o}}-\mathrm{Ys}$ ) in the in situ camera calibration bundle adjustment. These correlations may allow the parameters related to focal length and coordinates of principal point to absorb some inaccuracies connected to the direct estimation of the Xs, Ys and Zs. These correlations do not invalidate this study since the estimated IOP will be used to perform the experiment of the Integrated Sensor Orientation considering the operational conditions of the photogrammetric survey.

The parameters values and the profiles of radial lens distortions, reported in Table 2 and Figure 2 respectively, reveal a slight variation in the radial lens distortion obtained from in situ calibrations and camera certificate. This small variation can be considered as an expected result due to the effect of the photogrammetric refraction in the aerial images (Andrade, 1977).

\begin{tabular}{|c|c|c|c|}
\hline \multicolumn{4}{|c|}{ IOP from in situ camera calibration } \\
\hline $\mathbf{f}(\mathbf{m m})$ & 51.674 & $\sigma_{\mathrm{f}}(\mathbf{m m})$ & 0.009 \\
\hline $\mathbf{x}_{0}(\mathbf{m m})$ & 0.103 & $\sigma_{\mathrm{x} 0}(\mathrm{~mm})$ & 0.003 \\
\hline $\mathbf{y}_{\mathbf{0}}(\mathbf{m m})$ & 0.131 & $\sigma_{\mathrm{yo}}(\mathrm{mm})$ & 0.002 \\
\hline $\mathrm{k}_{1}\left(\mathrm{~mm}^{-2}\right)$ & $-1.441997 \mathrm{E}-05$ & $\sigma_{\mathrm{k} 1}\left(\mathrm{~mm}^{-2}\right)$ & 5.071053E-07 \\
\hline $\mathrm{k}_{2}\left(\mathrm{~mm}^{-4}\right)$ & $3.789018 \mathrm{E}-09$ & $\sigma_{\mathrm{k} 2}\left(\mathrm{~mm}^{-4}\right)$ & $4.910055 \mathrm{E}-10$ \\
\hline \multicolumn{4}{|c|}{ IOP from the camera manufacturer certificate } \\
\hline $\mathbf{f}(\mathbf{m m})$ & 51.695 & $\mathrm{k}_{1}\left(\mathrm{~mm}^{-2}\right)$ & $-1.4309 \mathrm{E}-05$ \\
\hline $\mathbf{x}_{\mathbf{0}}(\mathbf{m m})$ & 0.014 & $\mathbf{k}_{2}\left(\mathbf{m m}^{-4}\right)$ & 3.8639E-09 \\
\hline $\mathbf{y}_{\mathbf{0}}(\mathbf{m m})$ & 0.059 & & \\
\hline
\end{tabular}

$\left(\mathrm{k}_{1}, \mathrm{k}_{2}\right)=$ Radial lens distortion; $(\sigma)=$ Standard deviation;

Table 2. Estimated IOP from the in situ camera calibration and nominal IOP values from the camera manufacturer certificate

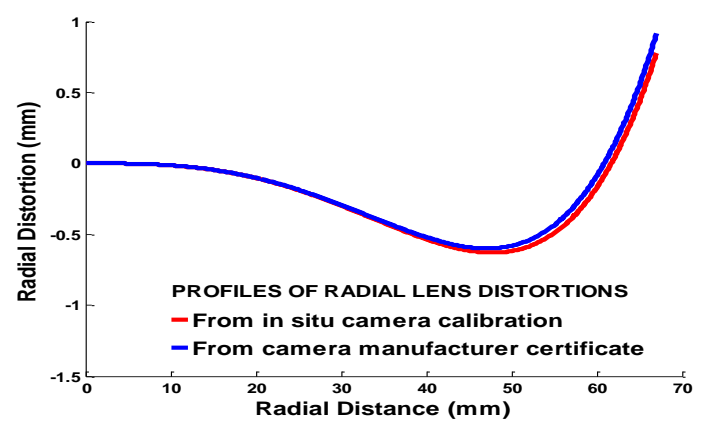

Figure 2. Profiles of radial lens distortions. 


\subsection{Integrated sensor orientation without control points}

As mentioned before, the main objective of this study is to verify the importance of the in situ camera calibration and Integrated Sensor Orientation to improve the accuracies of photogrammetric and Lidar datasets integration. For this purpose, two ISO experiments are performed. The first uses the IOP provided by the camera manufacturer certificate and in the second one, the IOP, estimated by the in situ self-camera calibration (reported in Table 2), were used. The values of EOPs from the direct sensor orientation were included in the bundle adjustment as additional observations by weight constraint. In the first experiment, the EOP values were weighted according to the nominal precisions (10 centimeters for the positions (Xs, Ys, Zs) and 18 seconds for $\omega$ and $\varphi$ and 29 seconds for $\chi$ ). The second experiment used the EOP precisions estimated in situ calibration, reported in Table 1 (2, 1, 14 centimeters for Xs, Ys, $\mathrm{Zs}$ and $11,4,7$ seconds for $\omega, \varphi, \chi$ respectively). Both ISO experiments use half of pixel $(0.003 \mathrm{~mm})$ for measurements precisions in image coordinates.

The entire photogrammetric images block, showed in Figure 1, is used in both experiments. It has 96 images distributed in 6 strips taken in opposite directions, 37 Lidar control points and 334 tie points over the overlap areas defined and measurement by automatic procedures using LPS software. The Lidar control Points measurements were conducted manually using monocular view. The thirty-seven Lidar Control Points are used as the check points. The horizontal and vertical discrepancies are computed by comparison of 3D coordinates obtained by 3D photogrammetric intersection and three Lidar planes intersection.

The main results obtained in two ISO experiments and the analyses of 3D discrepancies of the 3D coordinates of 37 Lidar Control Points are shown in Table 3 and 4 respectively. The obtained results from the two ISO experiments, reported in Tables 3 and 4, showed that the accuracies of the ISO experiment were improved significantly when the IOP values and precisions values of the EOP, estimated in situ camera calibration, were used; as can be seen by the values of posteriori variance of unit weight.

\begin{tabular}{|c|c|c|c|c|c|c|c|c|c|}
\hline \multicolumn{10}{|c|}{$\begin{array}{l}\text { RESIDUALS ANALYSIS OF TWO ISO } \\
\text { EXPERIMENTS }\end{array}$} \\
\hline \multicolumn{3}{|c|}{$\begin{array}{c}\text { RMSE of the } \\
\text { Residuals in image } \\
\text { coordinates (microns) }\end{array}$} & \multicolumn{6}{|c|}{$\begin{array}{l}\text { RMSE of the } \\
\text { residuals in EOPs } \\
\text { (centimeters and seconds) }\end{array}$} & \multirow[b]{2}{*}{$\sigma_{0}$} \\
\hline & $\mathbf{x}$ & $\mathbf{y}$ & $\mathbf{X s}$ & Ys & Zs & $\omega$ & $\varphi$ & $\chi$ & \\
\hline $\begin{array}{c}\text { IOP } \\
\text { Manufacturer }\end{array}$ & 3 & 4 & 79 & 73 & 6 & 184 & 198 & 61 & 17.991 \\
\hline $\begin{array}{l}\text { IOP } \\
\text { In situ }\end{array}$ & 2 & 2 & 0.4 & 0.1 & 11 & 11 & 1 & 3 & 1.001 \\
\hline \multicolumn{10}{|c|}{ Maximum residuals } \\
\hline $\begin{array}{c}\text { IOP } \\
\text { Manufacturer }\end{array}$ & 10 & 17 & 104 & 97 & 22 & 248 & 248 & 209 & \\
\hline $\begin{array}{l}\text { IOP } \\
\text { In situ }\end{array}$ & 8 & 14 & -1 & 0.1 & 34 & 20 & 3 & 8 & \\
\hline $\begin{array}{r}\mathbf{R N} \\
\left(\boldsymbol{\sigma}_{\mathbf{0}}\right)=\end{array}$ & & Roo & variar & nce & & Wo & & & \\
\hline
\end{tabular}

Table 3. Main results of the residuals analyses of the two ISO experiments

\begin{tabular}{|c|c|c|c|c|c|}
\hline \multicolumn{6}{|c|}{ CHECK POINTS DISCREPANCIES } \\
\hline & & DX & DY & DH & $\mathbf{D Z}$ \\
\hline \multirow{3}{*}{$\begin{array}{c}\text { IOP } \\
\text { Manufacturer }\end{array}$} & $\mu$ & 5.5 & 23.7 & 61.4 & 132.3 \\
\hline & RMSE & 51.8 & 49.8 & 71.8 & 141.6 \\
\hline & Max & 160.5 & 122.8 & 160.7 & 235.4 \\
\hline \multirow{3}{*}{$\begin{array}{c}\text { IOP } \\
\text { In situ }\end{array}$} & $\mu$ & 10.4 & 11.4 & 46.6 & 32.7 \\
\hline & RMSE & 38.6 & 35.4 & 52.4 & 47.5 \\
\hline & Max & 77.3 & 108.3 & 120.6 & 110.5 \\
\hline
\end{tabular}

RMSE = Root mean square error of the discrepancies $(\mathrm{cm})$; Max $=$ Maximum discrepancy $(\mathrm{cm})$ DH $=$ Horizontal discrepancy $(\mathrm{cm})$

Table 4. Results of discrepancies analysis performed in check points from two ISO experiments

A close look at Figures 3 and 4 reveals a small variation of the images' EOP, computed by direct sensor orientation, when the ISO experiment used IOP from in situ camera calibration. The majority of the residuals have values according to the a priori adopted precisions. However, the Zs coordinate residuals and Omega orientation residuals have values greater than their precisions that were estimated in the in situ camera calibration.

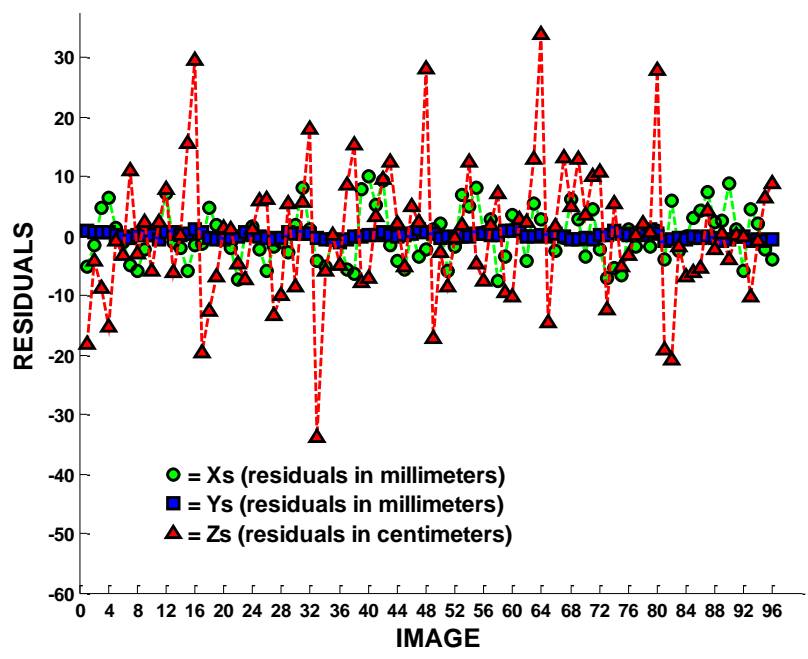

Figure 3. Residuals of the image positions obtained in the ISO experiment using IOP in situ camera calibration

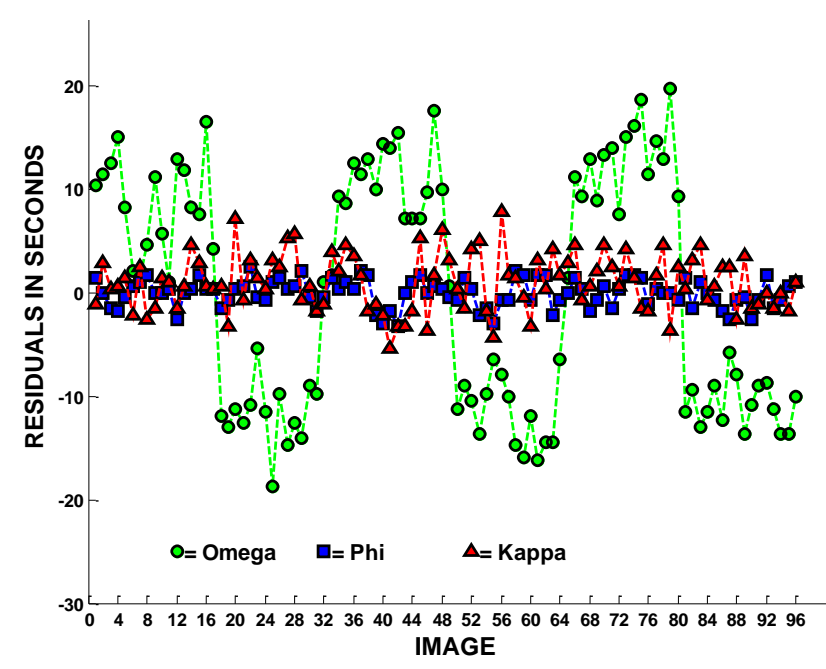

Figure 4: Residuals of the image orientations obtained in the ISO experiment using IOP in situ camera calibration. 
Considering the values of root mean square errors of the check point discrepancies, the photogrammetric 3D intersection increased around of $37 \%$ and $198 \%$ the horizontal and vertical accuracies respectively. Although the IOP from in situ camera calibration improved the ISO experiment, the horizontal and vertical accuracies, estimated by the RMSE of the 3D discrepancies, did not achieve the expected accuracies considered for this study.

Two GSD $(36 \mathrm{~cm})$ was the expected horizontal accuracy considered for this study. According to Vosselman and Maas (2010), the horizontal position accuracy of a control points, extracted from Lidar point cloud, can vary from 0.1 to 1.0 meters. For the expected vertical accuracy it was considered the vertical accuracy of the 3-D photogrammetric intersection, estimated based on the average flight height $(1.600 \mathrm{~m})$, the average baseline $(508 \mathrm{~m})$, the image measurement precision $(0.003 \mathrm{~mm})$, and the focal length $(51.7 \mathrm{~mm})$. Using the mathematic equation, shown in Alamús and Kornus (2008), the expected vertical accuracy is close to $41 \mathrm{~cm}$.

As can be seen in Table 4 and Figure 5, the values of horizontal and vertical discrepancies and theirs root mean square errors are greater than the expected accuracies. Additionally, there is a small vertical tendency not fixed by the IOP estimated the in situ camera calibration. These results indicate a presence of a systematic errors not modeled by the proposed methodology. These inaccuracies can be related to the quality of the Lidar control points computed by three Lidar planes intersection and how the LCPs inaccuracies affect the IOP estimation by proposed methodology. Moreover, the position of the image sub block inside of the entire image block can modify quality of the IOP estimation under flight conditions. Future studies will be conducted to verify these assumptions.

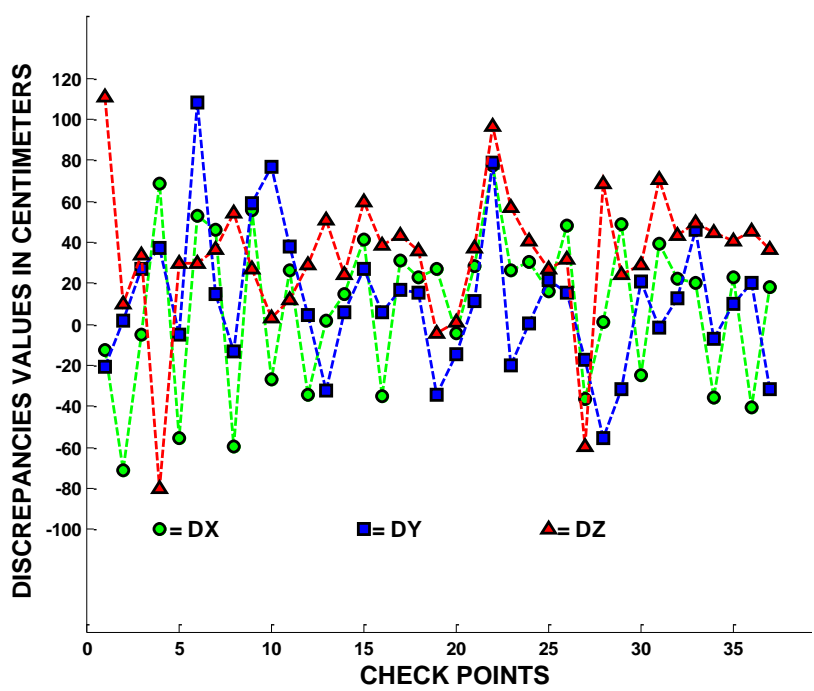

Figure 5. 3D discrepancies from the check points analyses obtained in the ISO experiment using IOP in situ camera calibration.

\section{CONCLUSION AND FUTURE WORK}

The paper presented an empirical study of the importance of the in situ camera calibration to perform the Integrated Sensor Orientation in order to increase the accuracies of the photogrammetric and LIDAR datasets integration. The Lidar dataset is used as a control of position information for the photogrammetric survey performed by the direct sensor orientation technology. Two ISO experiments without control points were performed. The first used the IOP provided by the photogrammetric company (camera manufacturer certificate) and in the second one, the IOP, estimated by the in situ camera calibration, were used. From the results of the performed experiments, the main conclusions are drawn:

(a) The horizontal and vertical accuracies of photogrammetric and Lidar datasets integration by photogrammetric procedure improved significantly when ISO approach were performed using IOP values and precisions values of the EOP, estimated in situ camera calibration; the values of root mean square errors of the Lidar check point discrepancies increased around of $37 \%$ and $198 \%$ the horizontal and vertical accuracies respectively;

(b) Although the IOP from in situ camera calibration improved the performance of the ISO experiment, the horizontal and vertical accuracies, estimated by the RMSE of the 3D discrepancies, did not achieve the expected accuracies considered in this study; there is a small vertical systematic tendency not fixed by the estimated IOP;

(c) Majority of the direct EOP residuals, obtained in ISO experiment using calibrated IOP, have values according to the a priori adopted precisions. However, some values of Zs coordinate residuals and Omega orientation residuals did not achieve this precision, causing the inaccuracies aforementioned;

(d) The in situ camera calibration using small sub block of images and three LCPs, proposed to estimate IOP under flight condition, achieved acceptable precisions; the estimated IOP values increased the accuracies of ISO experiment.

Future works will continue to verify whether the spatial position of the sub block of images, inside of the entire block, can change the performance of proposed methodology to increase the photogrammetric and Lidar datasets by ISO approach and 3D photogrammetric point intersection.

\section{ACKNOWLEDGMENTS}

We would like to thank the two Brazilian governmental agencies $\mathrm{CNPq}$ (The National Council for Scientific and Technologic Development) and CAPES (The Coordinating Agency for Advanced Training of High-Level Personnel) for their financial support of this research and Technology Institute for Development, LACTEC, UFPR, Brazil for providing the Lidar and image datasets.

\section{REFERENCES}

Alamús, R. AND KoRnUs, W., 2008. DMC geometry analysis and virtual image characterization. The Photogrammetric Record, 23 (124), 353-371. doi:10.1111/j.14779730.2008.00504.x

ANDRADE, J.B., 1977. Photogrammetric refraction. Ph.D. dissertation, The Ohio State Univ., Columbus, OH, USA, 1977.

Brown, D., 1971. Close range camera calibration, Photogrammetric Engineering, 37(8), 855-866.

Costa, F., A. L., Mitishita, E., Centeno, J., 2017. A Study of Integration Of Lidar And Photogrammetric Datasets by Indirect Georeferencing and In Situ Camera Calibration, International Journal of Image and Data Fusion. doi.org/10.1080/19479832.2017.1294625 
Cramer, M., AND D. Stallmann, 2001. OEEPE test on integrated sensor orientation - IFP results and experience, Proceedings of the OEEPE Workshop, Integrated Sensor Orientation, Hanover, Germany, 17-18 September.

CRAmer, M. AND Stallmann, D., 2002. System Calibration for Direct Georeferencing, International Archives of Photogrammetry, Remote Sensing and Spatial Information Sciences, 34(3/A), 79-84.

Delara, R., Mitishita, E., And Habib, A., 2004. Bundle adjustment of images from nonmetric CCD camera using LIDAR data as control points. International Archives for Photogrammetry, Remote Sensing and Spatial Information Sciences, 20 (B3), 13-18.

Habib, A., Ghanma, M., and Mitishita, E., 2004. Coregistration of photogrammetric and LIDAR data: methodology and case study. Revista Brasileira De Cartografia, 1, 56.

HabiB, A., GHANMA, M., AND KIM, E.M., 2005. LIDAR data for photogrammetric georeferencing. In: FIG Working Week 2005 and GSDI-8. April. Cairo, Egypt.

Heipke, C., K. Jacobsen, And H. Wegmann, 2002. Analysis of the Results of the OEEPE Test "Integrated Sensor Orientation", Integrated Sensor Orientation, Test Report and Workshop Proceedings (C. Heipke, K. Jacobsen, and H. Wegmann, editors), OEEPE Official Publication No. 43, 31-49.

Honkavaara, E., Ilves, R., JaAkKola, J., 2003. Practical Results of GPS/IMU/camera System Calibration. In: Proc. Of Workshop: Theory, Technology and Realities of Inertial/GPS/Sensor Orientation, ISPRS WG I/5, Barcelona, CD-ROM, $10 \mathrm{p}$.

JaCobsen, K., 2000. Combined Bundle Block Adjustment versus Sensor Orientation, ASPRS Annual Convention 2000, Washington D.C.
Jacobsen, K., Wegmann, H., 2002. Dependencies and problems of Direct Sensor Orientation. "Integrated Sensor Orientation", Integrated Sensor Orientation, Test Report and Workshop Proceedings (C. Heipke, K. Jacobsen, and $\mathrm{H}$. Wegmann, editors), OEEPE Official Publication No. 43, 73-84.

Kersting, A. P., 2011. Quality Assurance of Multi-Sensor Systems, PhD Thesis, University of Calgary, Department Of Geomatics Engineering, Calgary, Canada, 265 pages.

Mitishita, E. A., Habib, A., Centeno, J., Machado, A., Lay, J. AND Wong, C., 2008. Photogrammetric and LIDAR Data Integration Using the Centroid of a Rectangular Roof as a Control Point, The Photogrammetric Record, 23(121), pp. 1935, 2008. doi:10.1111/j.1477-9730.2008.00464.x

Vosselman, G. AND MAAS, H.-G., 2010. Airborne and terrestrial laser scanning. Scotland, UK: Whittles Publishing Dunbeath.

Wegmann, H., 2002. Image Orientation by Combined (A)AT with GPS and IMU. In: Proceedings of ISPRS Commission I Mid-term Symposium, Nov. 2002, Denver, Colorado, USA

Wildan, F., Aldino, R., AND AJI, P.P. 2011. Application of LIDAR technology for GCP determination in papua topographic mapping scale 1:50.000. In: 10th Annual Asian Conference \& Exhibition on Geospatial Information, Technology \& Applications. 17-19 October. Jakarta, Indonesia. 13

YASTIKLI, N., JACOBSEN K., 2005. Influence of System Calibration on Direct Sensor Orientation. Photogrammetric Engineering and Remote Sensing, Vol. 71 No 5, May 2005, 629-633. 